In Developments in Renewable Energies Offshore (1st ed.)

(9p.)

October 2020

Ed. Guedes Soares, C.

ISBN : 9781003134572

https://doi.org/10.1201/9781003134572-71

https://archimer.ifremer.fr/doc/00732/84413/

\title{
Blade root load variations on two scaled industrial tidal turbines
}

Slama M. ${ }^{1}$, Pinon G. ${ }^{1}$, Togneri M. ${ }^{2}$, Jump E. ${ }^{3}$, Gaurier Benoit ${ }^{4}$, Germain Gregory ${ }^{4}$, Represas F. ${ }^{5}$, Grande J. ${ }^{5}$, Nicolas E. ${ }^{6}$, Marcille J. ${ }^{6}$

\footnotetext{
${ }^{1}$ Normandie Univ, UNILEHAVRE, Le Havre, France

2 Swansea University, Bay Campus, Swansea, UK

3 Offshore Renewable Energy Catapult, Glasgow, Scotland

${ }^{4}$ IFREMER, Boulogne-sur-mer, France

${ }^{5}$ Magallanes Renovables, Redondela, Spain

${ }^{6}$ Sabella S.A., Quimper, France
}

\begin{abstract}
:
This experimental study investigates blade root load fluctuations for a single turbine immersed in a regular current flow with different ambient turbulence conditions. Two scaled models of industrial prototypes were tested: a 1:28 scale model of the three-bladed horizontal axis turbine of Magallanes Renovables and a 1:20 scale model of the five-bladed horizontal axis turbine of Sabella. The rotor loads were directly measured on the rotation axis with a thrust and torque sensor. The blade roots were equipped with a loadcell measuring two forces and three moments. The variations of the rotor loads (thrust and torque) and the blade root loads (edgewise bending moments and streamwise forces) are compared and the impact of the blade angular position is investigated. A comparison of both scaled models results with those of the IFREMER generic turbine is also presented. The results are anonymised in order to ensure confidentiality.
\end{abstract}




\section{INTRODUCTION}

Increasing the reliability of tidal energy converters is, with cost reduction, one of the two most important challenges in the coming decade for the tidal industry. Several projects were recently launched focusing on one or these two goals: Met-Certified (Scheijgrond et al. 2019), Marinet 2 Round-Robin tests (Gaurier et al. 2019), a H2020 project Real-Tide or a FCEInterreg programme called TIGER. The MONITOR project (Togneri et al. 2019), an Atlantic-Area Interreg program is one of them. It gathers academics, industries and research institutes focusing their effort on improving the blade reliability of such tidal energy converters. One Work-package is dedicated to laboratory testings and aims to characterise the fluc- tuating loads for a single turbine immersed in a regular current flow with different levels of turbulence or wave conditions. Two scaled pre-commercial prototypes are under test: Magallanes Renovables' ATIR tidal platform and the bottom mounted D12 turbine of Sabella; both companies being involved in the MONITOR partnership.

The ATIR is a floating $2 \mathrm{MW}$ device composed of a 3-bladed bi-rotor turbine with variable pitch mechanisms. A first full scale prototype was tested at the Ría de Vigo in Spain (Diaz-Dorado et al. 2020). The platform was then deployed at the European Marine Energy Centre (EMEC) in Scotland, during summer 2018.

Sabella's D12 is composed of a single rotor with 5 fixed (no-pitch) and symmetrical blades. The turbine 
diameter is $12 \mathrm{~m}$ for a power output of $500 \mathrm{~kW}$ and is scheduled for installation in 2021. Sabella's earlier D10 model operating on a similar design has already undergone two test deployments in 2015 and 2018.

An interesting feature is that these two rotors were mounted on the same nacelle and motor-assembly, and tested under the same flow conditions in a single experimental facility. Load variations obtained for three turbulence intensities $(1.5 \%, 3 \%$ and $15 \%)$ and for one upstream velocity $\left(U_{\infty}=1.2 \mathrm{~m} / \mathrm{s}\right)$ are presented. More results, particularly the torque and thrust coefficients of the two scaled turbines for several incoming velocities, are available in Pinon et al. (2019) and Slama et al. (2020). In fact, tidal energy converters subjected to real in-situ flows can undergo high ambient turbulence and severe wave conditions (Milne et al. 2016, Togneri et al. 2017, Guo et al. 2018). Such load variations can affect the turbines reliability, particularly their blades, and should be taken into account at the design stage. Therefore, after the presentation of the experimental set-up, the phaseaveraged rotor loads (thrust and torque) and blade root loads (edgewise bending moments and streamwise forces), for each turbine, are presented in Section 3. A comparison with the results of IFREMER's generic turbine (Gaurier et al. 2019) is then presented in Section 4.

\section{EXPERIMENTAL SET-UP}

\subsection{Flume tank description and experimental configurations}

All the trials were carried out in the IFREMER wave and current flume tank in Boulogne-sur-mer (see Fig. 1 and Gaurier et al. (2018)). The hub of the scaled turbine was set at a height of $1 \mathrm{~m}$ below the free surface. The flow conditions were monitored with a bidimensional Laser Doppler Velocimeter (LDV) and an Acoustic Doppler Velocimeter (ADV).

\subsection{Upstream flow conditions}

The ambient turbulence intensity $I_{\infty}$ generated in the flume tank can be regulated using flow straighteners placed at the inlet of the working section. It goes from $I_{\infty} \approx 1.5 \%$ when both a grid and a honeycomb are used, to $I_{\infty} \approx 3 \%$ when only the honeycomb is used and to a higher value of $I_{\infty} \approx 15 \%$ when removing all the flow straighteners. The bi-dimensional turbulence intensity $I_{\infty}$ is here defined as:

$$
I_{\infty}=100 \sqrt{\frac{1 / 2\left(\sigma_{u_{\infty}}^{2}+\sigma_{v_{\infty}}^{2}\right)}{\bar{u}_{\infty}^{2}+\bar{v}_{\infty}^{2}}}
$$

where $\sigma_{u_{\infty}}$ and $\sigma_{v_{\infty}}$ are the standard deviations of the velocity components $u_{\infty}$ and $v_{\infty}$ of the upstream velocity $\boldsymbol{u}_{\infty}\left(\boldsymbol{u}_{\infty}=u_{\infty} \boldsymbol{e}_{\boldsymbol{x}}+v_{\infty} \boldsymbol{e}_{\boldsymbol{y}}+w_{\infty} \boldsymbol{e}_{\boldsymbol{z}}\right)$. The overbar denotes the time average. Much more turbulence characterisation on the present experimental campaigns can be found in Gaurier et al. (2020) and Slama et al. (2020). Lastly, the mean upstream axial velocity range used in these trials goes from $U_{\infty}=$ $0.8 \mathrm{~m} / \mathrm{s}$ to $U_{\infty}=1.4 \mathrm{~m} / \mathrm{s}$. Only the results obtained at $U_{\infty}=1.2 \mathrm{~m} / \mathrm{s}$ are presented in this paper.

\subsection{Turbine models description}

The scale of the ATIR and the D12 models, presented in Figure 2, are 1:28 and 1:20 respectively. The models are based on the existing IFREMER generic turbine, a three-bladed device with a horizontal axis, used in previous works (Gaurier et al. 2019). The rotors were mounted on the same instrumented hub, only the blades and the careenage for the nacelle were changed in order to adapt the turbine to the industrial geometries. Model parameters, such as the hub radius and the blade lengths and materials, are detailed in Table 1.

Table 1: Turbine model parameters.

\begin{tabular}{lcc}
\hline Description & ATIR & D12 \\
\hline Scale & $1: 28$ & $1: 20$ \\
Rotor Radius $R[\mathrm{~mm}]$ & 338 & 300 \\
Hub Radius [mm] & 55 & 96 \\
Hub length [mm] & 720 & 720 \\
Number of blades & 3 & 5 \\
Blade length [mm] & 283 & 204 \\
Direction of rotation & $\mathrm{C}$ & $\mathrm{CC}$ \\
Blade material & $\mathrm{A}$ & $\mathrm{CF}$ \\
\hline
\end{tabular}

C: clockwise; CC: counter-clockwise; $\mathrm{CF}$ : Carbon fibre; A: Aluminium.

The rotor of the scaled models is connected to a motor-gearbox assembly consisting of a gearbox, a DC motor, a ballast load and a motor speed control unit (Mycek et al. 2014, Gaurier et al. 2019), providing an active rotor speed control. Due to the reduced scale, the pitch of the blades is fixed in each case. The Tip Speed Ratio (TSR) is the ratio of the tip velocity of the blade to the upstream flow velocity:

$$
T S R=\frac{\omega_{x} \times R}{U_{\infty}}
$$

where $\omega_{x}$ is the axial rotation speed. In this study, the TSR varies from 0 to 8 for the ATIR model and from 0 to 6 for the D12 turbine.

The torque and thrust are directly measured on the rotation axis with a waterproof transducer. For the three-bladed ATIR model, each blade root is equipped with a load-cell (see Fig. 3) measuring two forces (along $\boldsymbol{e}_{\boldsymbol{x} i}$ and $\boldsymbol{e}_{\boldsymbol{y} \boldsymbol{i}}$, with $i=1$ to 3 ) and three moments (around $e_{x i}$ (edgewise bending moment), $e_{y i}$ (flapwise bending moment) and $\boldsymbol{e}_{\boldsymbol{z} i}$ (pitching moment)). 


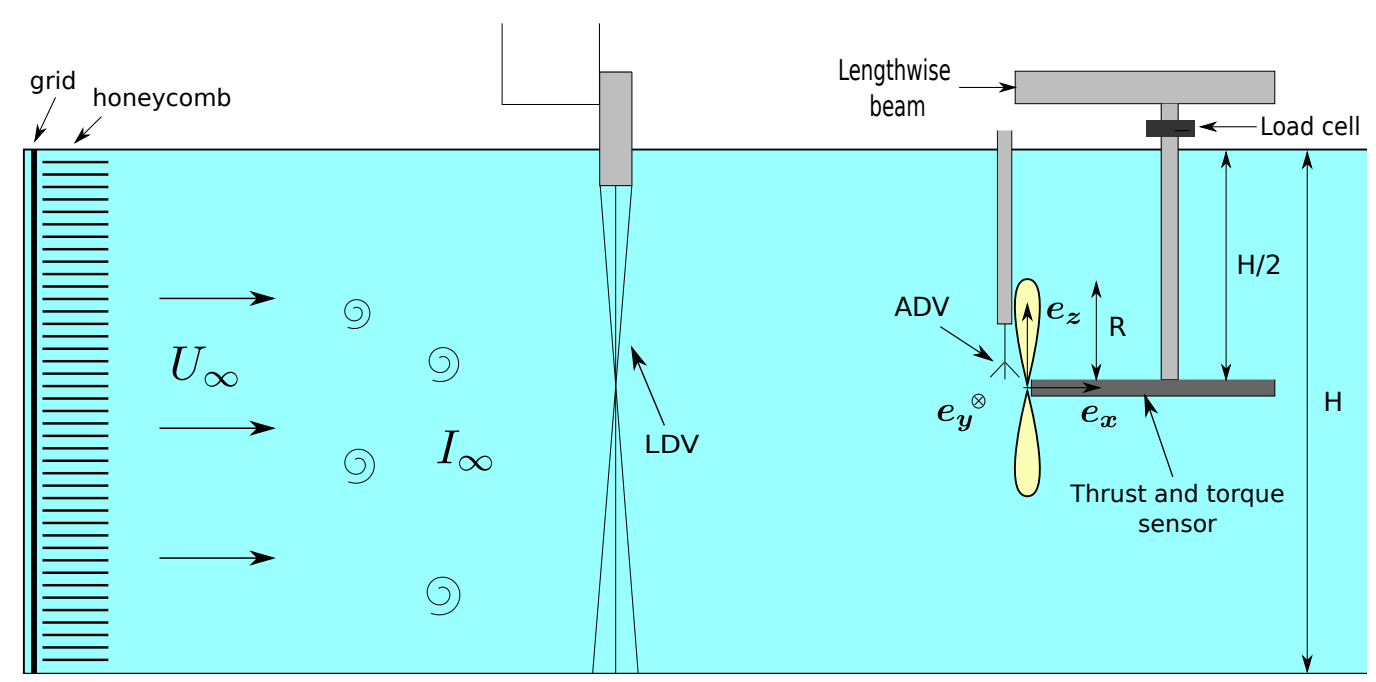

Figure 1: Schematic side view of the experimental set-up in the $(18 \mathrm{~m} \times 4 \mathrm{~m} \times 2 \mathrm{~m})$ wave and current flume tank at Ifremer.

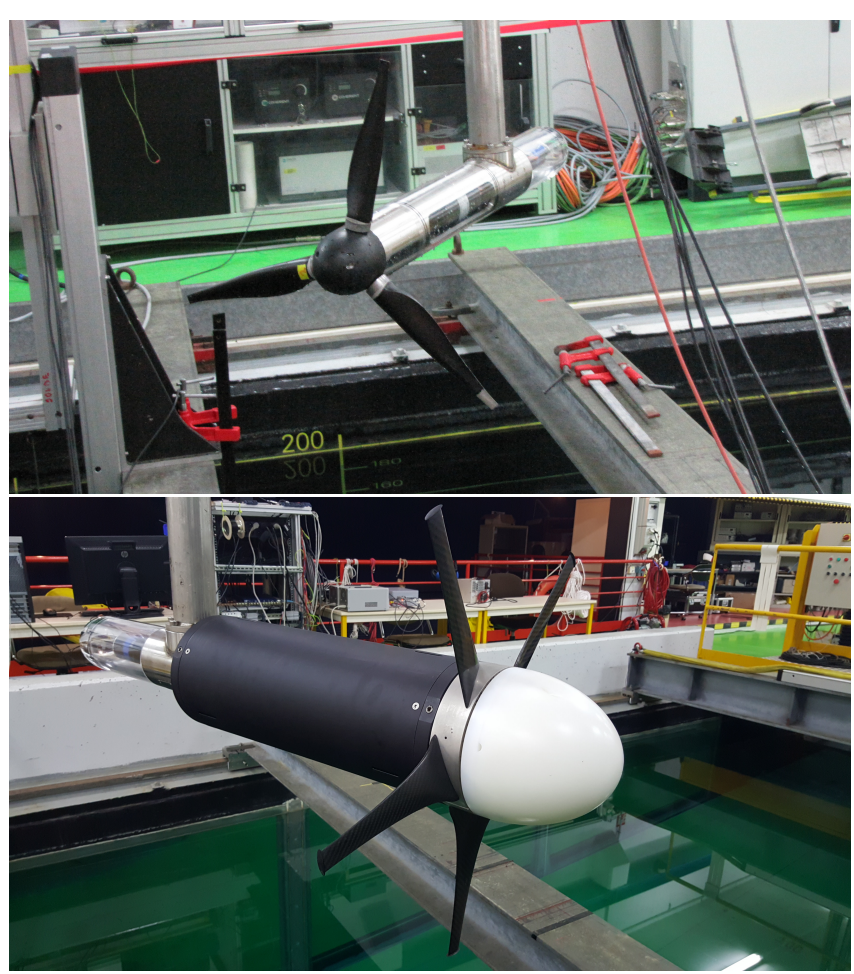

Figure 2: Pictures of the scaled models: Magallanes Renovables' ATIR (top) and Sabella's D12 turbine (bottom).

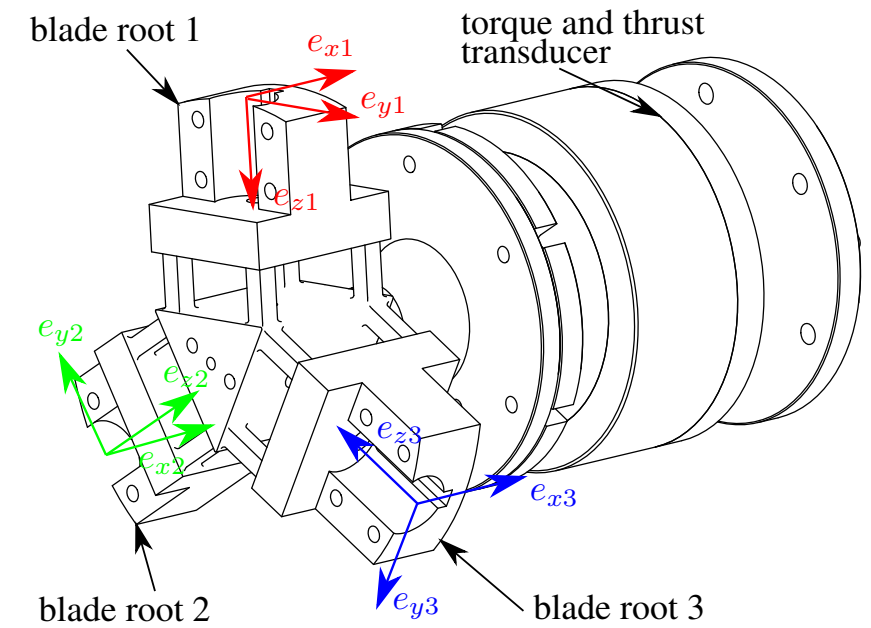

Figure 3: The blade root load-cells with the three coordinate systems and the torque and thrust transducer.
Since there are only three load-cells on the existing instrumented hub, three blade supports were manufactured in order to adapt the five-bladed D12 model to the IFREMER device: one was used to support one blade and the other two support two blades. Thus, for the D12 scaled turbine, the blade root loads are studied only for one blade.

More details about the experimental set-up can be found in Pinon et al. (2019) and Slama et al. (2020).

\section{ROTOR AND BLADE ROOT LOADS}

The rotor angular position is evaluated using the projection of the blade root edgewise forces. From the estimation of the angular position, the centred load measurements are split into angular sectors with a width of $1^{\circ}$. By calculating the averages and the standard deviations of any load $Z$ for each angular sector, two quantities can be defined: the phase-averaged $Z$, denoted by $\widetilde{Z}^{\theta}$; the phase-standard deviation of $Z$, denoted by $\sigma_{\theta}(Z)$ (see Fig. 9 for more details).

As the industrial turbine performances are confidential, blade root loads (axial forces $F_{x i}$ and edgewise bending moments $M_{x i}$ ), torque $Q$ and thrust $T$ results are divided by reference values (denoted by the subscripts $r e f$ ), which are the time averages of the loads for a specific case. The anonymised phaseaveraged forces and moments are defined by:

$$
\begin{aligned}
& \widetilde{\mathcal{Q}}^{\theta}=\frac{\widetilde{Q}^{\theta}}{\overline{Q_{\text {ref }}}} ; \widetilde{\mathcal{M}}_{x i}^{\theta}=\frac{{\widetilde{M_{x i}}}^{\theta}}{\overline{M_{x i \mathrm{ref}}}} ; \widetilde{\mathcal{M}}^{\theta}=\frac{\widetilde{\sum M_{x i}}}{\sum \bar{M}_{x i \mathrm{ref}}} ; \\
& \widetilde{\mathcal{T}}^{\theta}=\frac{\widetilde{T}^{\theta}}{\overline{T_{\text {ref }}}} ; \widetilde{\mathcal{F}}_{x i}^{\theta}=\frac{\widetilde{F_{x i}}}{\overline{F_{x i \mathrm{ref}}}} ; \widetilde{\mathcal{F}}^{\theta}=\frac{\widetilde{\sum F_{x i}}}{\sum \overline{F_{x i \mathrm{ref}}}} ;
\end{aligned}
$$

where $i$ is the blade index. The phase-standard deviations are anonymised similarly. The velocity cases for the reference values were chosen in order to have the highest and closest Reynolds numbers between the scaled models. Thus, the selected veloci- 
ties for anonymising the data are $U_{\infty}=1.3 \mathrm{~m} / \mathrm{s}$ for the ATIR and $1.4 \mathrm{~m} / \mathrm{s}$ for the D12. The corresponding Reynolds numbers $R e_{\infty}$ are respectively $4.39 \times 10^{5}$ and $4.20 \times 10^{5}$. The reference values were then chosen for $I_{\infty}=1.5 \%$ and at the TSR for which the power coefficient reached its maximum (see Slama et al. (2020)). For the rest of the paper, it is reminded here that the presented results are all obtained for an incoming velocity of $U_{\infty}=1.2 \mathrm{~m} / \mathrm{s}$ for all the turbines.

\subsection{Magallanes Renovables' ATIR turbine}

Figures 4 and 5 show the variations (in percent) of the phase-averaged blade root loads at TSR $\approx 5$, for $I_{\infty}=1.5 \%$ (Fig. 4 and top panels of Fig. 5) and $I_{\infty}=15 \%$ (bottom panels of Fig. 5). Left frames present the axial forces for each blade and right plots correspond to the edgewise bending moments. In Figure 4 , when the rotor angular position equals to $0^{\circ}$, the blade 1 is vertical at the top. In Figure 5, the angular position is adjusted for blades 2 and 3. At the lowest turbulence intensity, the three curves of the axial forces $\widetilde{\mathcal{F}}_{x i}{ }^{\theta}$ superimpose well and reach two maxima (and minima) which are about $180^{\circ}$ apart, when the blades are nearly vertical (horizontal). At $I_{\infty}=15 \%$, the shape of the curves changes: the variations are greater in the $150^{\circ}-240^{\circ}$ sector and the minimum is reached in the $300^{\circ}-330^{\circ}$ sector. The increase of the ambient turbulence also induces an increase of the blade root load fluctuations which are multiplied by approximately 3 . Regarding the phaseaveraged bending moments $\widetilde{\mathcal{M}}_{x i} \theta$, the curves superimpose but there are more differences, particularly for blade 3 which presents slightly larger variations (Fig. 5). It can be observed that the bending moments variations are larger than those of $\widetilde{\mathcal{F}}_{x i}{ }^{\theta}$. This is due to the large contribution of the weight force to the edgewise bending moments. This contribution varies with the blade angular position: it reaches a maximum when the blade angle equals to $90^{\circ}$ and a minimum at $270^{\circ}$. The same evolution can be seen for the phaseaveraged moments, particularly at $I_{\infty}=1.5 \%$ (top right panel of Fig. 5). For the highest turbulence intensity, the variations increase by $5 \%$ in the $60^{\circ}-120^{\circ}$ and the $210^{\circ}-240^{\circ}$ sectors with respect to its lowest turbulence intensity counterparts.

Figure 6 presents a comparison of the phaseaveraged rotor loads variations with those of the sum of the blade root loads, for both turbulence intensities. It can be observed, on the top left panel, that the thrust variations at $I_{\infty}=1.5 \%$ are small (they range between $-0.6 \%$ and $0.5 \%$ ) and are very close to those of the sum of the axial blade root forces. Similarly to Payne et al. (2018), three lobes spaced about $120^{\circ}$ apart can be identified. They are due to the passage of the three blades. On the top right frame, the variations of the sum of the edgewise root bending moments are compared to the torque ones. In this case, they are slightly larger and range between $-2 \%$ and $2 \%$. Three lobes are also visible but the curves superimpose only for one lobe. In the $60^{\circ}-140^{\circ}$ region, the torque fluctuates more than $\widetilde{\mathcal{M}}^{\theta}$ whereas it is the opposite in the $300^{\circ}-0^{\circ}$ sector. Furthermore, there is a lag of about $15^{\circ}$ between the lobes of the left and right top panels. At $I_{\infty}=15 \%$ (bottom panels), there is an increase of the rotor and blade root load fluctuations. Indeed, $\widetilde{\mathcal{T}}^{\theta}$ and $\widetilde{\mathcal{F}}^{\theta}$ variations range from $-2 \%$ to $2 \%$ and those of $\widetilde{\mathcal{Q}}^{\theta}$ and $\widetilde{\mathcal{M}}^{\theta}$ from $-8 \%$ to $8 \%$. This corresponds to a multiplication by 3 to 4 compared to the results for $I_{\infty}=1.5 \%$. The increase of the turbulence intensity also leads to a modification of the curves shapes. The variations due to the turbulence hide the impact of the blades passage and the three lobes are no longer visible on the two bottom frames.

\subsection{Sabella's D12 turbine}

Figure 7 presents the variations of the phase-averaged rotor thrust and the phase-averaged sum of the blade root forces, for the lowest turbulence intensity. As the ATIR model, the D12 thrust variations are small and close to those of $\widetilde{\mathcal{F}}^{\theta}$. Five small lobes, due to the five blades passage, are visible.

Figure 8 shows the edgewise root bending moment on blade 1 at $I_{\infty}=15 \%$. The shape of the curve is similar but reversed compared to the ATIR results. This is due to the difference in the direction of rotation of both scaled turbines. These two last figures (Fig. 7 and Fig. 8) comfort the previous analysis already made on the ATIR turbine in subsec. 3.1. As a short summary on this topic, the maxima and minima of the loads as function of the angular direction could most probably be attributed to: the number of blades, the weight of the blades (edgewise bending moment), and possibly some modifications in the incoming flow properties with increased ambient turbulence.

\section{COMPARISON OF THE LOAD VARIATIONS}

The load variations were evaluated for the whole TSR range, for each turbine and for the three turbulence intensities. In order to synthesize the results, only the maxima of the absolute value of phase-averages $\left(\bullet^{\theta}\right)$ and the phase-standard deviations $\left(\sigma_{\theta}(\cdot)\right)$ of the blade root loads are reported in this section. An example of the selected quantities is shown in Figure 9 for the ATIR model. The results obtained for the ATIR and D12 scaled models, presented in Figures 10-13, are compared to those obtained for IFREMER's generic turbine (Gaurier et al. 2019) at $U_{\infty}=1.0 \mathrm{~m} / \mathrm{s}$.

It can be seen in Figures 10 and 11 that the evolutions of the axial force phase-averages and phasestandard deviations are similar for the three turbines. Both the averages and the standard deviations increase with the ambient turbulence intensity, $\sigma_{\theta}\left(\mathcal{F}_{x 1}\right)$ 

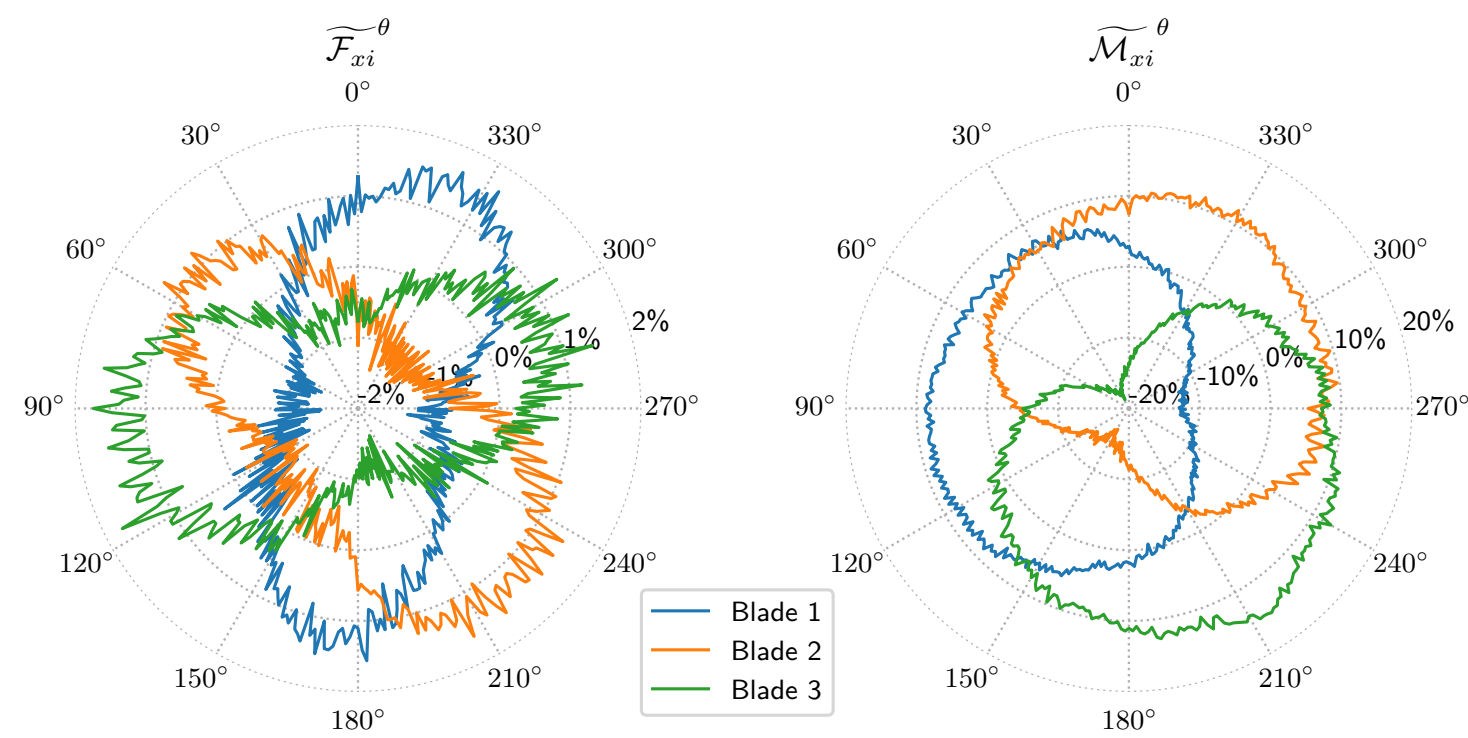

Figure 4: Variations of the phase-averaged blade root loads with the rotor angular position for the ATIR model $\left(I_{\infty}=1.5 \%-U_{\infty}=1.2\right.$ $\mathrm{m} / \mathrm{s}-\mathrm{TSR} \approx 5$ ). Left panel: axial forces, right panel: edgewise moments.

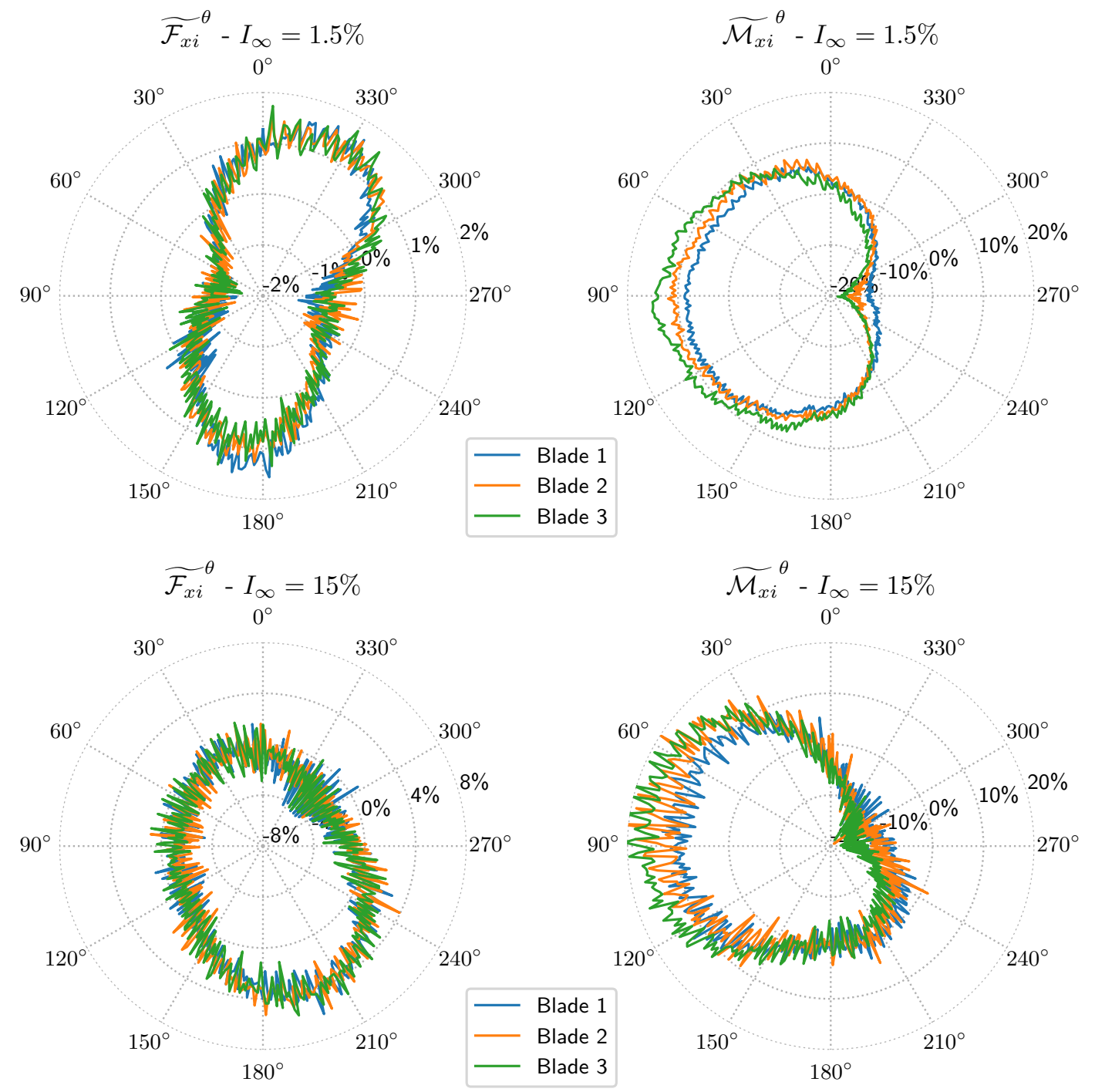

Figure 5: Variations of the phase-averaged blade root loads with the rotor angular position for the ATIR model $\left(U_{\infty}=1.2 \mathrm{~m} / \mathrm{s}-\right.$ $\mathrm{TSR} \approx 5)$, for two ambient turbulence intensity cases. The angular position is adjusted for blades 2 and $3\left(\theta+120^{\circ}\right.$ and $\theta-120^{\circ}$ respectively).

being particularly impacted. In this last Figure 11, the results for the intermediate turbulence intensity value $\left(I_{\infty}=3 \%\right)$ are clearly distinguishable from the lower intensity value. For these two quantities, it seems that the driving parameter is the ambient turbulence value and that the turbine design (blade shape, solidity) does not have an influence.

The bending moments variations are also affected 

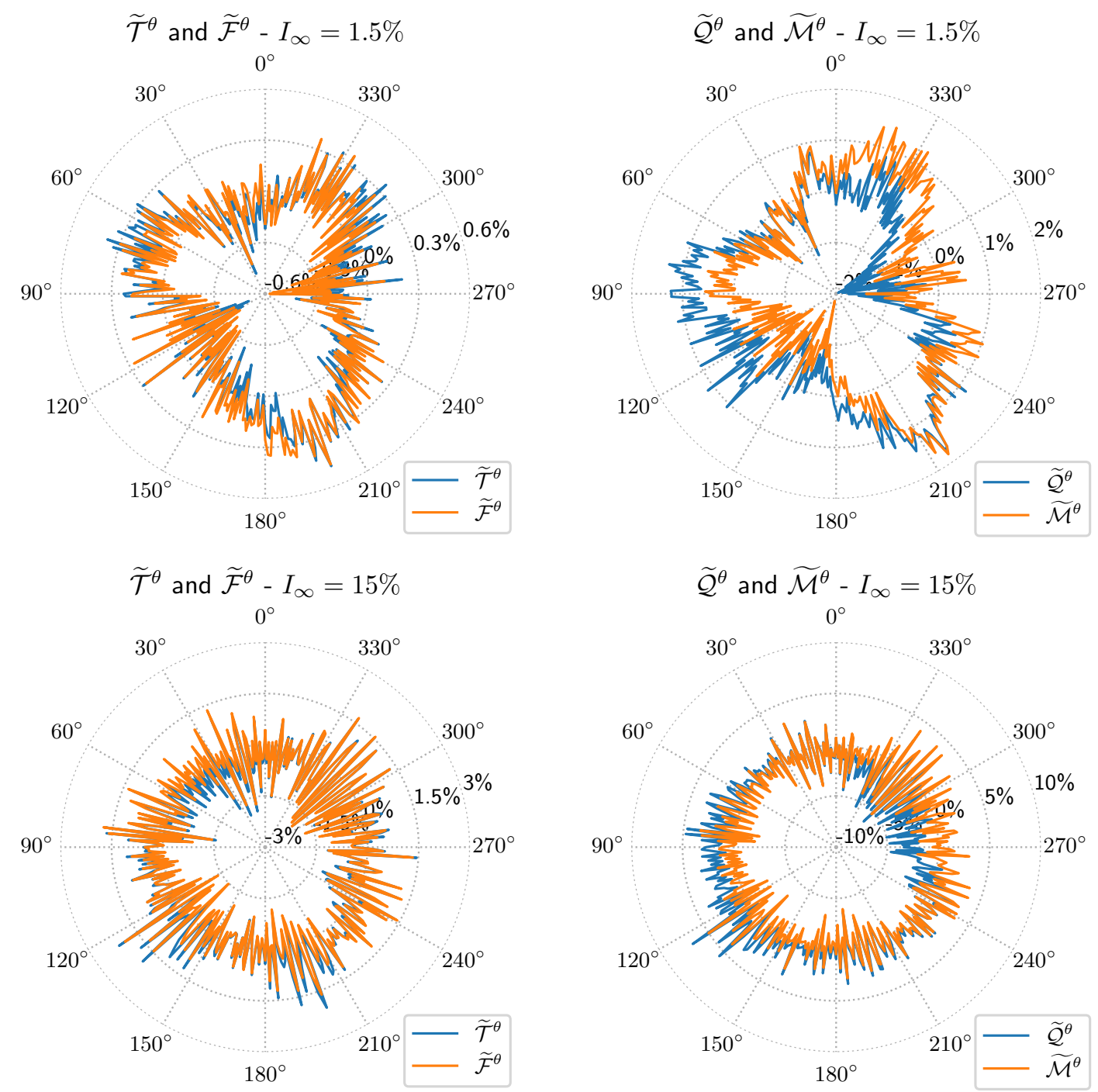

Figure 6: Comparison of the phase-averaged rotor and blade root loads variations for the ATIR model $\left(U_{\infty}=1.2 \mathrm{~m} / \mathrm{s}-\mathrm{TSR} \approx 5\right)$, for two ambient turbulence intensity cases. Left panels: rotor thrust and sum of the axial blade root forces; right panel: rotor torque and sum of the edgewise blade root bending moments.

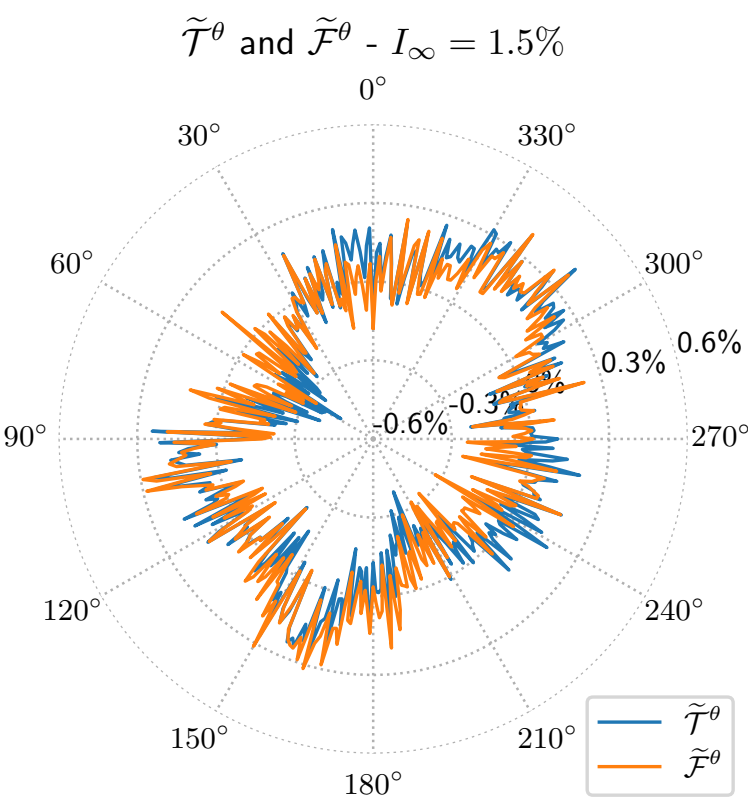

Figure 7: Comparison of the variations of the phase-averaged rotor thrust and sum of the axial blade root forces for the D12 model $\left(U_{\infty}=1.2 \mathrm{~m} / \mathrm{s}-\mathrm{TSR} \approx 5-I_{\infty}=1.5 \%\right)$.

by the ambient turbulence but, as already observed in Figure 5 for the ATIR model, this is not the only im-

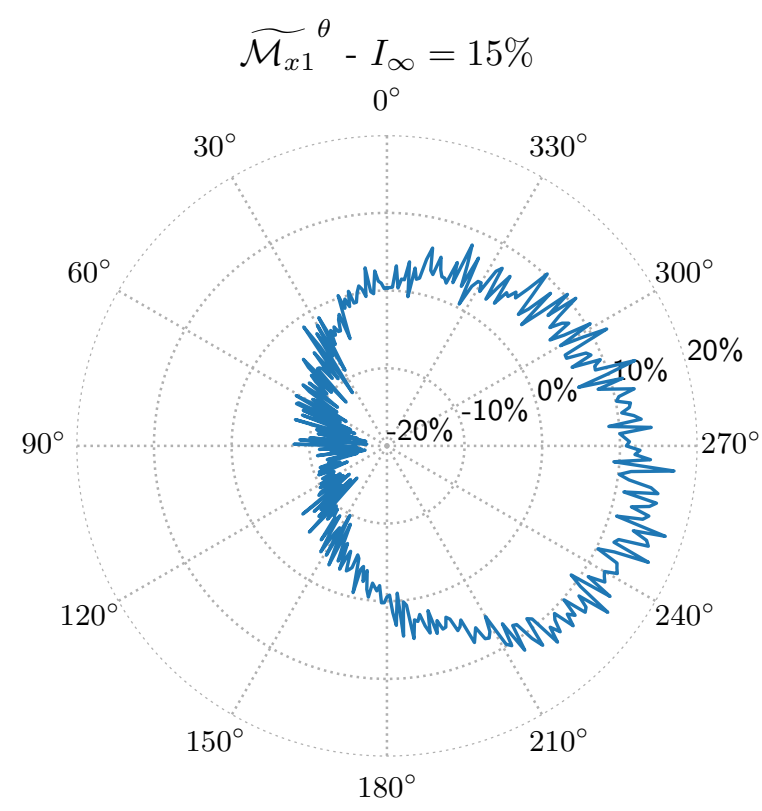

Figure 8: Variations of the phase-averaged edgewise root bending moment for blade 1 for the D12 model $\left(I_{\infty}=15 \%-U_{\infty}=\right.$ $1.2 \mathrm{~m} / \mathrm{s}-\mathrm{TSR} \approx 5)$.

portant parameter. In fact, from Figure 12, one can observe that the major difference is due to the turbine 


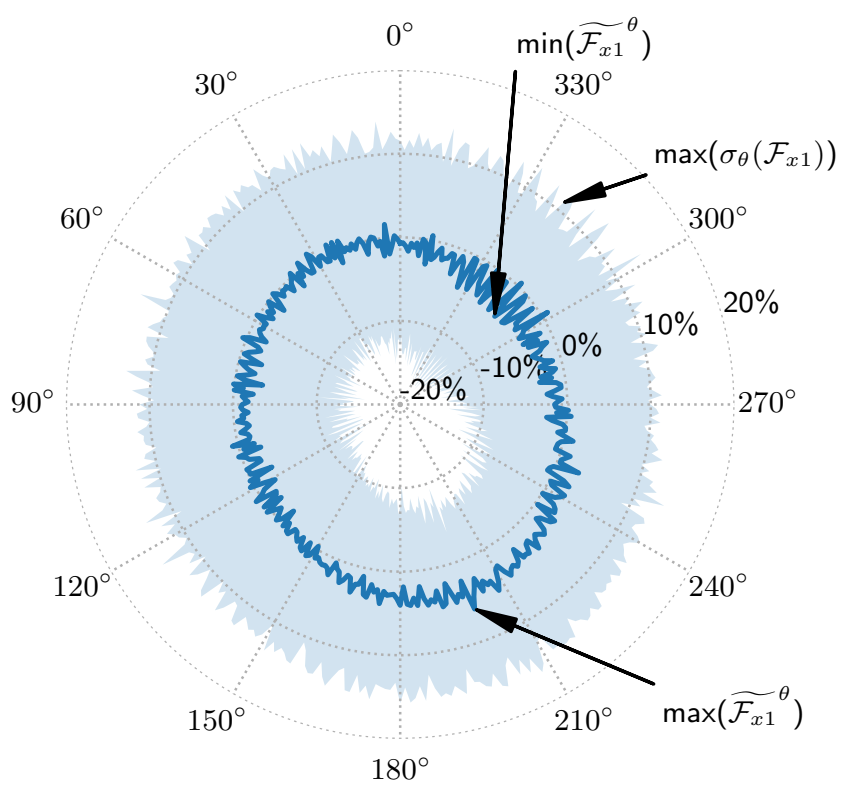

Figure 9: Variations of the phase-averaged axial force on blade 1 for the ATIR model $\left(I_{\infty}=15 \%-U_{\infty}=1.2 \mathrm{~m} / \mathrm{s}-\mathrm{TSR} \approx 5\right)$. The filled area corresponds to the confidence interval with the phase-standard deviation.

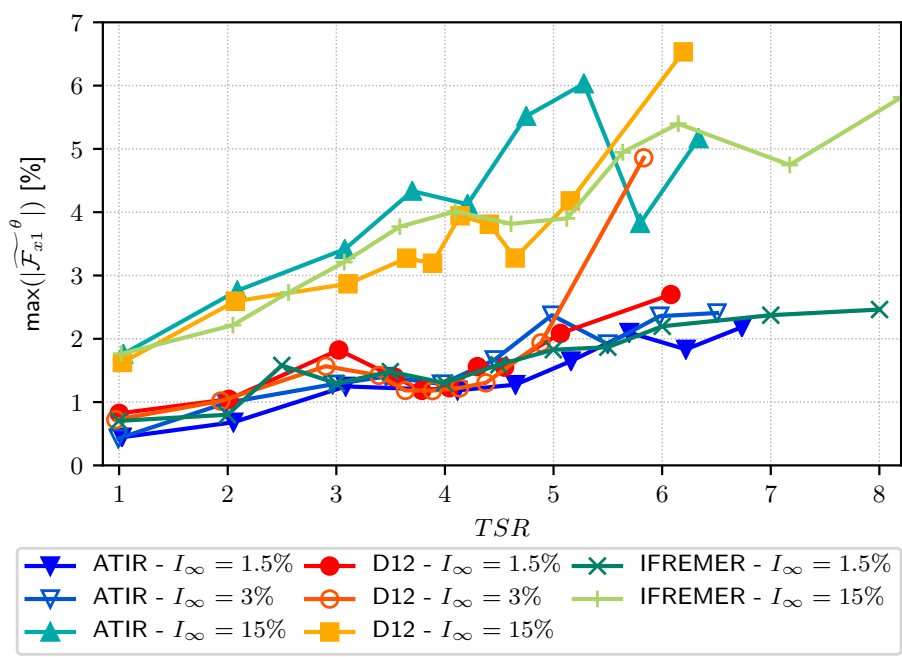

Figure 10: Maxima of the phase-averaged axial force on blade 1 with respect to the TSR.

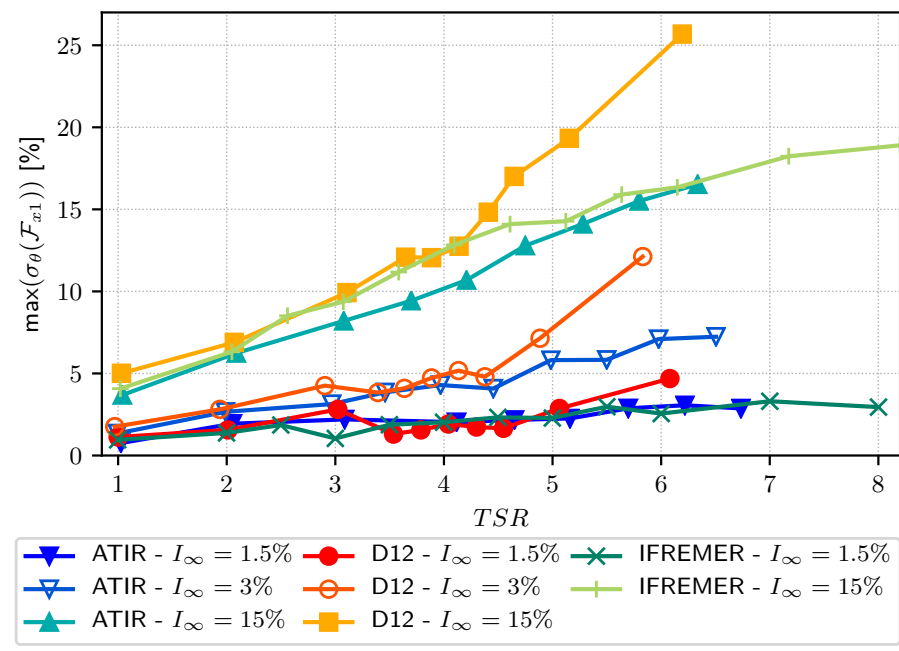

Figure 11: Maxima of the phase-standard deviation of the axial force on blade 1 with respect to the TSR.

the blade is originating. Differences in blade's weight have a major influence on $\max \left(\left|\widetilde{\mathcal{M}}_{x 1}{ }^{\theta}\right|\right)$. Secondly, to

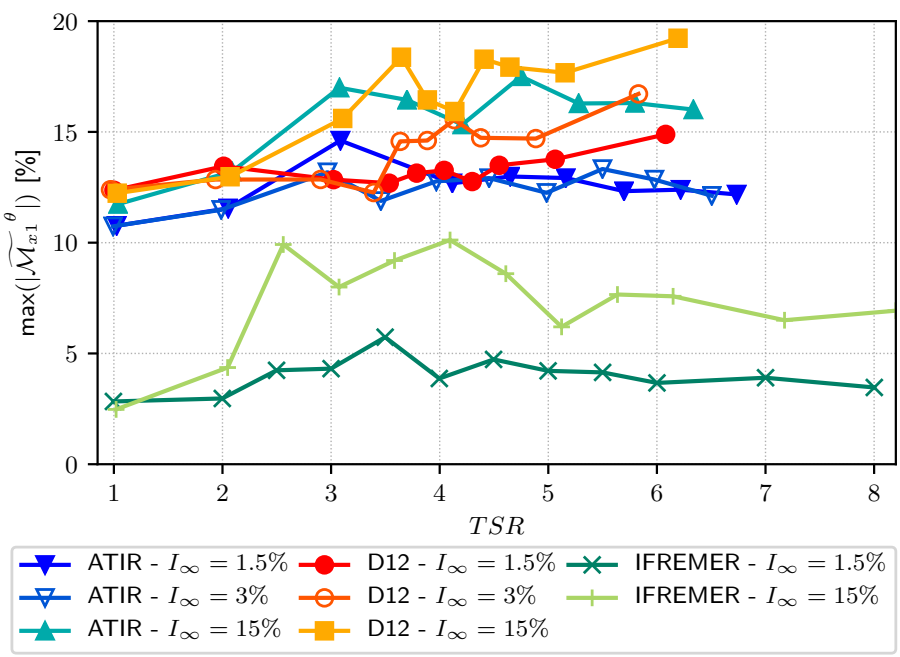

Figure 12: Maxima of the phase-averaged bending moment on blade 1 with respect to the TSR.

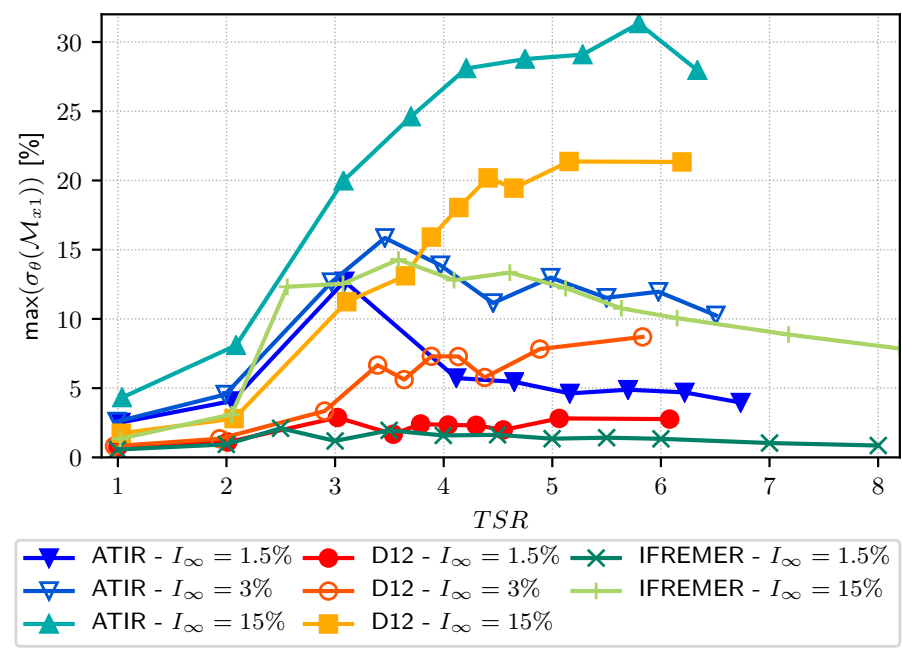

Figure 13: Maxima of the phase-standard deviation of the bending moment on blade 1 with respect to the TSR.

a lower extent, the turbulence intensity only slightly increases quantity. However, compared to the other parameters, the TSR does not have a major importance and the results are quasi unchanged with respect to the TSR value.

On the contrary, the most complex response is obtained for the $\max \left(\left|\sigma_{\theta}\left(\mathcal{M}_{x 1}\right)\right|\right)$ presented in Figure 13. In fact, the turbulence intensity clearly have a large influence on this quantity but the blade shape also have a major influence. As a matter of fact, in the operating range of TSR, $\max \left(\left|\sigma_{\theta}\left(\mathcal{M}_{x 1}\right)\right|\right)$ increase from $\approx 2 \%$ to $\approx 13 \%$ from the lowest to the highest turbulence intensity, for IFREMER's turbine. A similar behaviour from $\approx 2.5 \%$ to $\approx 18 \%$ is observable for the D12 turbine, and from $\approx 5 \%$ to $\approx 27 \%$ for the ATIR turbine. The rotor design here has a major influence which is logical as the torque, and hence $\mathcal{M}_{x 1}$, highly depend on the hydrodynamic performance of the profile.

Additionally, from Figures 10, 12 and 13, increasing TSR largely increases the load variations. This observation is completely validated for both the phaseaverage and the phase-standard deviation of $\mathcal{F}_{x 1}$ as the absolute quantities (non-anonymised quantities) 
also increase with the TSR. Nevertheless, this needs further validation of the phase-standard deviation of $\mathcal{M}_{x 1}$ as the torque decreases with increasing TSR passed the optimal range. However operating the turbine in over-speed regions may deteriorate more rapidly the turbine and lead to increased security coefficients during the design. Finally, from Figures 12 and 13, the rotor design (blade number, profile, solidity, camber and mass) also has a large impact.

\section{CONCLUSIONS}

Scale models of two industrial tidal turbine prototypes were studied: a 1:28 scale model of the three-bladed turbine of Magallanes Renovables and a 1:20 scale model of the five-bladed device of Sabella. The rotor and blade root load variations were analysed for three ambient turbulence conditions. The study pointed out that the blade root load variations are 3 to 7 times larger than the rotor ones. It is therefore important to measure and evaluate correctly the former in order to improve the device reliability. It was also seen that the blade root load variations strongly depend on the blade angular position.

A comparison of the load variations of both scale models with the results obtained for the opengeometry tidal turbine of IFREMER showed that the axial force variations are mainly impacted by the ambient turbulence whereas the edgewise bending moments are also largely affected by the rotor design. Finally, it is reminded here that these two studied quantities (phase-average and phase-standard deviation) actually add themselves leading to fluctuating loads at the order of 45 to $50 \%$ even in the operating range for the highest ambient turbulence level. For floating turbines, but not limited to, wave induced velocity oscillation may further increase these load fluctuations. Such configuration were already tested and will be analysed in a near future.

\section{ACKNOWLEDGEMENTS}

This project is co-financed by the European Regional Development Fund (ERDF) through the Interreg Atlantic Area Programme, via the MONITOR project. This work was also supported in part by the ERDF and the Normandy Regional Council via programs such as NEPTUNE, SEMARIN and DIADEMAR. This work is part of the RealTide project which received funding from the European Union's Horizon 2020 research and innovation programme under grant agreement $n^{\circ} 727689$.

\section{REFERENCES}

Diaz-Dorado, E., C. Carrillo, J. Cidrás, D. Román, \& J. Grande (2020). Testing and modeling of the marine tidal current platform atir. Under review at Renewable Energy.
Gaurier, B., C. Carlier, G. Germain, G. Pinon, \& E. Rivoalen (2020). Three tidal turbines in interaction: An experimental study of turbulence intensity effects on wakes and turbine performance. Renewable Energy 148, 1150 - 1164.

Gaurier, B., G. Germain, J.-V. Facq, \& T. Bacchetti (2018). Wave and current flume tank of ifremer at boulogne-sur-mer. description of the facility and its equipment. Report, FRANCE.

Gaurier, B., G. Germain, \& G. Pinon (2019). How to correctly measure turbulent upstream flow for marine current turbine performances evaluation? In Advances in Renewable Energies Offshore: Proceedings of the 3rd International Conference on Renewable Energies Offshore (RENEW 2018), October 8-10, 2018, Lisbon, Portugal. 1st Edition. Carlos Guedes Soares (Ed.). ISBN 978-1-138-58535-5. Modelling tidal currents. pp.23-30 (Taylor \& Francis Group).

Gaurier, B., S. Ordonnez, \& G. Germain (2019, Sep 1-Sep 6). First round of MaRINET 2 tidal energy round robin tests: combined wave and current tests,. In D. Vicinanza (Ed.), Proceedings of the Thirteenth European Wave and Tidal Energy Conference, Universitá degli Studi della Campania "Luigi Vanvitelli", Italy. EWTEC. ISSN: 2309-1983.

Guo, X., J. Yang, Z. Gao, T. Moan, \& H. Lu (2018). The surface wave effects on the performance and the loading of a tidal turbine. Ocean Engineering 156, 120 - 134.

Milne, I., A. Day, R. Sharma, \& R. Flay (2016). The characterisation of the hydrodynamic loads on tidal turbines due to turbulence. Renewable and Sustainable Energy Reviews 56, $851-864$.

Mycek, P., B. Gaurier, G. Germain, G. Pinon, \& E. Rivoalen (2014). Experimental study of the turbulence intensity effects on marine current turbines behaviour. part I: One single turbine. Renewable Energy 66(0), 729 - 746.

Payne, G. S., T. Stallard, R. Martinez, \& T. Bruce (2018). Variation of loads on a three-bladed horizontal axis tidal turbine with frequency and blade position. Journal of Fluids and Structures 83, $156-170$.

Pinon, G., C. E. Hadi, M. Slama, J. N. no, P. Mansilla, E. Nicolas, J. Marcille, J.-V. Facq, I. Belarbi, B. Gaurier, G. Germain, A. Pacheco, \& M. Togneri (2019, Sep 1-Sep 6). Influence of turbulence and wave flow conditions on different scaled tidal turbines. In D. Vicinanza (Ed.), Proceedings of the Thirteenth European Wave and Tidal Energy Conference, Universitá degli Studi della Campania "Luigi Vanvitelli", Italy. EWTEC. ISSN: 2309-1983.

Scheijgrond, P., A. Southall, C. Bittencourt, P. Davies, P. Mathys, G. Germain, \& M. Geertzen (2019). Advencing IEC standardization and certification for tidal energy converters. In D. Vicinanza (Ed.), Proceedings of the Thirteenth European Wave and Tidal Energy Conference, Universitá degli Studi della Campania "Luigi Vanvitelli", Italy. EWTEC. ISSN: 2309-1983.

Slama, M., G. Pinon, C. E. Hadi, M. Togneri, B. Gaurier, G. Germain, J.-V. Facq, J. N. no, P. Mansilla, E. Nicolas, J. Marcille, $\&$ A. Pacheco (2020). Turbine design dependency to turbulence: an experimental study of three scaled tidal turbines. submitted to Ocean Engineering.

Togneri, M., E. Buck, A. MacLeod, E. Nicolas, J. Nuño, M. O'Connor, A. Pacheco, G. Pinon, \& I. Masters (2019, Sep 1-Sep 6). Multi-model analysis of tidal turbine reliability. In D. Vicinanza (Ed.), Proceedings of the Thirteenth European Wave and Tidal Energy Conference, Universitá degli Studi della Campania "Luigi Vanvitelli", Italy. EWTEC. ISSN: 2309-1983.

Togneri, M., M. Lewis, S. Neill, \& I. Masters (2017). Comparison of ADCP observations and 3D model simulations of turbulence at a tidal energy site. Renewable Energy 114, 273 282. Wave and Tidal Resource Characterization. 